\title{
RECOMENDAÇÃO ALTERNATIVA DE CALCÁRIO PARA SOLOS ALTAMENTE TAMPONADOS DO EXTREMO SUL DO BRASIL
}

\author{
ALTERNATIVE LIMING RECOMMENDATION FOR HIGHLY \\ BUFFERED SOILS OF SOUTHERN BRAZIL
}

\author{
Jaime Antonio de Almeida ${ }^{1}$ Paulo Roberto Ernani ${ }^{2}$ Kátia Cilene Maçaneiro ${ }^{3}$
}

\section{RESUMO}

A recomendação de calcário nos estados do extremo sul do Brasil (RS e SC) objetiva elevar o pH do solo até 6,0 para a maioria das culturas. No entanto, para solos altamente tamponados, que contenham bastante matéria orgânica, esse valor de pH pode ser bem menor. O presente trabalho objetivou avaliar as modificações químicas em vários parâmetros de solo relacionados com a acidez, ocasionadas pela elevação do $\mathrm{pH}$ até 5,2 , bem como comparar métodos de recomendação de calagem e quantidades necessárias para atingir esse $\mathrm{pH}$ relativamente às atuais recomendações. Utilizaram-se 28 (vinte e oito) solos ácidos de Santa Catarina, aos quais se aplicou calcário, em 1984, a fim de obterem-se as curvas de neutralização da acidez. Após cinco meses de incubação, determinaram-se os valores de $\mathrm{pH}, \mathrm{Ca}^{2+}+$ $\mathrm{Mg}^{2+}, \mathrm{K}^{+}, \mathrm{Na}^{+}$e $\mathrm{Al}{ }^{3+}$ trocáveis e de $(\mathrm{H}+\mathrm{Al})$ e, a partir desses valores, calcularam-se a soma de bases (S), CTC efetiva, CTC a pH 7,0, índice de saturação por bases (V\%), e índice de saturação por alumínio ( $m \%)$. Para diagnosticar a necessidade de calcário para elevar o pH a 5,2 compararam-se os métodos do $A l^{3+}, H+A l$, Saturação por Bases, SMP, e a combinação de $A l^{3+}$ mais M.O. A elevação do $\mathrm{pH}-\mathrm{H}_{2} \mathrm{O}$ para 5,2 diminuiu o $\mathrm{Al}^{3+}, \mathrm{na}$ média dos solos, de 4,24 para 0,67 $\mathrm{cmol}_{\mathrm{c}} \mathrm{kg}^{-1}$, o $\mathrm{H}+\mathrm{Al}$ de 13,04 para $8,74 \mathrm{cmol}_{c} \mathrm{~kg}^{-1}$ e a $\mathrm{m \%}$ de 52 para $8 \%$, e aumentou o $\mathrm{Ca}+$ $\mathrm{Mg}$ de 3,61 para 8,38 $\mathrm{cmol}_{c} \mathrm{~kg}^{-1}$ e a V\% de 24 para $50 \%$. Todos os métodos foram eficientes para recomendar calagem até $\mathrm{pH}$ $\mathrm{H}_{2} \mathrm{O} 5,2$, porém o $\mathrm{Al}+\mathrm{M}$.O. foi o mais preciso e o saturação por bases o menos eficiente. A elevação do pH para 5,2 ao invés de 5,5 ou 6,0 resultaria numa economia de calcário de, respectivamente, 1,4 e 3,1t ha ${ }^{-1}$. Quando consideram-se somente os solos altamente tamponados, com pH SMP inferior a 5,0, a economia de calcário seria de 3,0 e 7,0t ha $a^{-1}$ respectivamente, podendo atingir até 5,0 e 10,6t ha em solos com pH SMP 4,4.

Palavras-chave: calagem, $\mathrm{pH}$, métodos de recomendação de calcário, solos tamponados.

\section{SUMMARY}

Lime recommendation for Rio Grande do Sul (RS) and Santa Catarina (SC) States in Brazil aims to raise soil water pH up to 6.0 to most crops. For highly buffered soils containing high organic matter content, this $\mathrm{pH}$ value may be lower. This study was carried out to evaluate the magnitude of changes on soil parameter values related to acidity due to increases on soil water pH to 5.2, as well as to compare analytical methods for lime recommendation, and lime requirement rates to attain this $\mathrm{pH}$ value relatively to the actual recommendations to raise soil pH to 5.5 or 6.0. Twenty-eight soils from Santa Catarina State were limed in 1984 with increasing rates of calcium carbonate in order to get the acidity neutralization curves. After 5 months of incubation, soil $\mathrm{pH}$, exchangeable $\mathrm{Ca}^{2+}+\mathrm{Mg}^{2+}, \mathrm{K}^{+}, \mathrm{Na}^{+}$and $\mathrm{Al}^{3+}$, and $(H+A l)$ were determined. From these data the sum of base (S), effective CEC, CEC at pH 7.0, percentage base saturation (V\%), and $A l^{3+}$ saturation on effective CEC (m\%) were calculated. Four analytical methods $\left(A l^{3+}, H+A l, S M P\right.$, and base saturation), in addition to the sum of $A l^{3+}$ and organic matter $(O M)$, were compared to determine lime requirement to raise soil $\mathrm{pH}$ to 5.2. The increase of soil water $\mathrm{pH}$ to 5.2 decreased $\mathrm{Al}^{3+}$ from 4.24 to $0.67 \mathrm{cmol}_{c} \mathrm{~kg}^{-1}, \mathrm{H}+\mathrm{Al}$ from 13.04 to $8.74 \mathrm{cmol}_{c} \mathrm{~kg}^{-1}$ and $(\% \mathrm{~m})$ from 52 to $8 \%$, and increased $\mathrm{Ca}+\mathrm{Mg}$ from 3.61 to $8.38 \mathrm{cmol}_{c} \mathrm{~kg}^{-1}$ and V\% from 24 to $50 \%$. All analytical methods were efficient for lime recommendation to $\mathrm{pH} 5.2$, however, the combination of $\mathrm{Al}^{3+}$ and $\mathrm{OM}$ was the most efficient and base saturation the least efficient. To increase soil water $\mathrm{pH}$ to 5.2 rather than to 5.5 or 6.0 would result in an limestone economy of 1.4 and 3.0t ha ${ }^{-1}$, respectively. For the highly buffered soils, with pH-SMP equal or below 5.0, the economy would be still larger, respectively of 3.0 and $7.0 \mathrm{t} \mathrm{ha} \mathrm{h}^{-1}$, which could attain up to 5.0 and 10.6t ha $\mathrm{a}^{-1}$ for soils with $\mathrm{pH}-\mathrm{SMP}$ of 4.4 .

Key words: liming, pH, lime requirement methods, buffered soils.

\footnotetext{
${ }^{1}$ Engenheiro Agrônomo, Doutor, Professor do Departamento de Solos, Universidade do Estado de Santa Catarina (UDESC), CP. 281, 88520-000, Lages (SC). Bolsista de Pesquisa do CNPq. Autor para correspondência. Email: a2jaa@ cav.udesc.br.

2 Engenheiro Agrônomo, PhD., Professor da UDESC. Bolsista de Pesquisa do CNPq.

${ }^{3}$ Aluno do Curso de Agronomia da UDESC, bolsista de Iniciação Científica do CNPq/PIBIC. Recebido para publicação em 08.10.98. Aprovado em 03.02.99
} 


\section{INTRODUÇÃO}

Grande parte dos solos do extremo sul do Brasil são muito ácidos, deficientes em fósforo e apresentam toxidez de alumínio aos vegetais. Nas regiões com elevada altitude, predominam solos de textura argilosa ou muito argilosa, com altos teores de matéria orgânica, o que lhes confere um elevado tamponamento (ERNANI \& ALMEIDA, 1986). Em muitos desses solos, o pH SMP varia de 4,6 a 4,0, e, portanto, requerem de 11 a $15 \mathrm{t}^{-1}$ de calcário (PRNT 100\%) para elevar o $\mathrm{pH}-\mathrm{H}_{2} \mathrm{O}$ a 5,5 , e de 20 a $29 \mathrm{t} \mathrm{ha}^{-1}$ para elevá-lo a 6,5 (COMISSÃO, 1995).

O custo da aplicação dessas altas doses de calcário, mesmo para elevar o $\mathrm{pH}$ a 5,5, é elevado e economicamente inviável para muitos agricultores, pelo menos no início do processo produtivo. Por falta de recursos financeiros, esses produtores ou não aplicam essas doses ou aplicam-nas em quantidades inferiores, sem nenhum critério técnico, o que pode limitar o rendimento das culturas. Mesmo começando o processo produtivo com valores de $\mathrm{pH}$ inferiores aos que proporcionam os rendimentos máximos, é interessante dar alternativas aos produtores para obter maior chance de lucros em suas culturas. Com o passar dos anos, espera-se uma capitalização dos mesmos, que assim poderão adquirir calcário e elevar o $\mathrm{pH}$ do solo para patamares mais altos.

Os maiores prejuízos às plantas em solos ácidos são ocasionados pela presença de quantidades tóxicas de $\mathrm{Al}$ e de $\mathrm{Mn}$, e pela deficiência de $\mathrm{Ca}$ e/ou $\mathrm{Mg}$. Em baixos valores de $\mathrm{pH}$ do solo, a disponibilidade de $\mathrm{P}$ e de MO, assim como, a atividade microorgânica, são também negativamente afetados, porém assumem menor importância, pois esses problemas podem ser superados pela aplicação de maiores quantidades desses nutrientes e de N. A aplicação de $\mathrm{Ca}$ e $\mathrm{Mg}$ também é resolvida pela aplicação de doses baixas de calcário dolomítico, ao redor de 2 a $3 \mathrm{t} / \mathrm{ha}^{-1}$. Os maiores problemas nos solos ácidos, resumem-se, portanto, à fitotoxicidade de $\mathrm{Al}$ e de Mn.

Vários trabalhos de pesquisa, entretanto, têm demonstrado que os maiores incrementos ocasionados pela calagem no rendimento de várias culturas ocorrem até em torno de $\mathrm{pH}$ 5,5 (MASCARENHAS, 1983; SIQUEIRA, 1989; QUAGGIO, 1989; ERNANI et al., 1998), onde os níveis de Al trocável já são muito baixos ou inexistentes. Para solos com altos teores de matéria orgânica, essa faixa de $\mathrm{pH}$ pode ser ainda menor, principalmente se os valores de $\mathrm{P}$ e $\mathrm{K}$ forem altos (ERNANI et al., 1997).
O presente trabalho objetivou avaliar as principais modificações químicas causadas em vários solos catarinenses pela elevação do $\mathrm{pH}$ em água a 5,2 , bem como avaliar métodos de recomendação quantitativa de calcário para atingir esse valor de $\mathrm{pH}$.

\section{MATERIAL E MÉTODOS}

Utilizaram-se 28 (vinte e oito) solos ácidos catarinenses, coletados na camada superficial de $0-20 \mathrm{~cm}$ de profundidade. A classificação, o local de coleta e algumas características físicas e químicas desses solos estão na tabela 1. Outras informações sobre os mesmos podem ser obtidas no trabalho de ERNANI \& ALMEIDA (1986).

Em unidades experimentais de 2,0kg (base seca) de cada um desses solos, aplicaram-se, em 1984, sete doses crescentes de calcário, correspondentes, em t/ha, a $0 ; 0,25 ; 0,50 ; 0,75 ; 1,0 ; 1,25$ e 1,50 vezes o número de $\mathrm{cmol}_{\mathrm{c}} \mathrm{kg}^{-1} \mathrm{de} \mathrm{H}+\mathrm{Al}$, a fim de obterem-se as curvas de neutralização da acidez. $\mathrm{O}$ calcário foi obtido pela mistura de $\mathrm{CaCO}_{3}$ com $\mathrm{MgCO}_{3}$, puros e finamente moídos, na relação 3:1, em peso, e as doses foram corrigidas para PRNT $100 \%$. Após a homogeneização completa, os solos foram umedecidos até aproximadamente $80 \%$ da capacidade de campo e acondicionados em sacos plásticos, que foram abertos e revolvidos a cada trinta dias, durante 150 dias. Após esse período, coletaram-se amostras em todas as unidades experimentais e nelas determinaram-se os valores de $\mathrm{pH}$ $\mathrm{H}_{2} \mathrm{O}$ e de $\mathrm{pH}$ em solução de $\mathrm{CaCl}_{2} 0,01 \mathrm{M}(\mathrm{pH}-$ $\mathrm{CaCl}_{2}$ ), ambos na relação solo/solvente de 1:1. Determinaram-se também $\mathrm{Ca}^{2+}+\mathrm{Mg}^{2+}, \mathrm{K}^{+}, \mathrm{Na}^{+}$e $\mathrm{Al}^{3+}$ trocáveis e de $(\mathrm{H}+\mathrm{Al})$, e a partir desses valores, calcularam-se a soma de bases (S), CTC efetiva, CTC a pH 7,0, índice de saturação por bases (V\%) e índice de saturação por alumínio (m\%). Nas amostras que não receberam calcário, determinaram-se também os teores de argila e de matéria orgânica. Os valores desses parâmetros no $\mathrm{pH}$ 5,2 foram obtidos a partir das equações de regressão das curvas de neutralização.

$\mathrm{O} \mathrm{Al}^{3+}$ e o $\mathrm{Ca}^{2+}+\mathrm{Mg}^{2+}$ foram extraídos com solução de cloreto de potássio $1 \mathrm{~mol} \ell^{-1}$ e determinados por titulometria de neutralização e complexometria, com $\mathrm{NaOH}$ e EDTA respectivamente; o $\mathrm{H}$ + Al foi extraído com solução de acetato de cálcio $0,5 \mathrm{~mol} \ell^{-1}, \mathrm{pH} 7,0$, na relação solo-solução $1: 20$, e determinado através de titulometria de neutralização com $\mathrm{NaOH}$; o $\mathrm{Na}^{+}$e o $\mathrm{K}^{+}$foram extraídos com solução de acetato de amônio $1 \mathrm{~mol} \ell^{-1}$ e determinados por espectrofotometria de emissão; e a matéria orgânica e o pH-SMP foram determinados de acordo com TEDESCO et al. (1985). 
Para estimar a quantidade de calcário necessária para elevar o $\mathrm{pH}-\mathrm{H}_{2} \mathrm{O}$ dos solos para 5,2, testaram-se os métodos do alumínio trocável $\left(\mathrm{Al}^{3+}\right)$, hidrogênio mais alumínio $(\mathrm{H}+\mathrm{Al})$, saturação por bases e SMP, além da combinação dos valores de $\mathrm{Al}^{3+}$ mais M.O. A origem e o princípio desses métodos estão descritos com detalhes no trabalho de ERNANI \& ALMEIDA (1986). A necessidade de calcário fornecida pelo método do $\mathrm{Al}+\mathrm{M}$.O. foi obtida através de análise de regressão múltipla (stepwise ORIGIN 3.5 - Scientific and Technical Graphics in Windows), relacionando a necessidade de calcário por incubação com os valores de $\mathrm{Al}+$ M.O. dos solos.

\section{RESULTADOS E DISCUSSÃO}

A elevação do $\mathrm{pH}-\mathrm{H}_{2} \mathrm{O}$ para 5,2 diminuiu substancialmente os valores de alumínio nos solos. $\mathrm{O}$ alumínio trocável $\left(\mathrm{Al}^{3+}\right)$ diminuiu, na média dos

Tabela 1 - Caracterização taxonômica e valores de alguns parâmetros químicos dos solos utilizados ${ }^{(1)}$.

\begin{tabular}{|c|c|c|c|c|c|c|c|c|c|}
\hline Solo ${ }^{(2)}$ & Clas. & Unid Taxonômica & Argila & M.O. & pH SMP & $\mathrm{pH}-\mathrm{H}_{2} \mathrm{O}$ & $\mathrm{pH}-\mathrm{CaCl}_{2}$ & $S^{(3)}$ & CTC \\
\hline & & & \multicolumn{3}{|c|}{----- $\mathrm{g} \mathrm{kg}^{-1}$} & & & \multicolumn{2}{|c|}{--- $\mathrm{cmol}_{\mathrm{C}} \mathrm{kg}^{-1}$} \\
\hline 01 & $\mathrm{CH}$ & Lages & 410 & 60,1 & 4,60 & 4,45 & 3,70 & 1,3 & 14,0 \\
\hline 02 & $\mathrm{LBH}$ & Vacaria & 680 & 54,8 & 4,55 & 4,50 & 3,80 & 2,0 & 14,6 \\
\hline 03 & TB & Vacaria & 630 & 63,4 & 4,95 & 4,60 & 3,90 & 2,6 & 15,0 \\
\hline 06 & LRH & Durox & 710 & - & 4,60 & 4,50 & 3,90 & 2,6 & 23,4 \\
\hline 10 & LRH & Palma Sola & 710 & 73,4 & 4,55 & 4,60 & 3,80 & 1,7 & 18,3 \\
\hline 13 & LR & Erexim & 690 & 51,5 & 4,55 & 4,40 & 3,85 & 1,9 & 14,6 \\
\hline 14 & TB & Catanduvas & 750 & 74,8 & 4,60 & 4,35 & 3,85 & 5,4 & 20,0 \\
\hline 16 & LRH & Durox & 750 & 74,6 & 4,60 & 4,55 & 3,85 & 3,4 & 19,0 \\
\hline 17 & $\mathrm{CH}$ & Volta Grande & 310 & 59,0 & 4,45 & 3,95 & 3,60 & 2,3 & 14,1 \\
\hline 19 & TB & Vacaria & 620 & 65,8 & 4,70 & 4,80 & 4,05 & 6,0 & 19,2 \\
\hline 22 & LE & Canoinhas & 480 & 53,8 & 4,70 & 4,45 & 3,80 & 1,1 & 13,2 \\
\hline 23 & PE & Içara & 330 & 25,0 & 5,45 & 5,00 & 4,20 & 3,4 & 8,8 \\
\hline 24 & $\mathrm{CH}$ & Rancho Grande & 560 & 52,0 & 4,60 & 4,80 & 4,10 & 6,9 & 19,7 \\
\hline 28 & $\mathrm{PE}$ & Içara & 230 & 19,3 & 5,65 & 4,65 & 4,00 & 1,6 & 6,2 \\
\hline 29 & PV & Morro da Fumaça & 330 & 43,1 & 5,40 & 4,70 & 4,20 & 4,9 & 11,1 \\
\hline 30 & PV & Ibirama & 190 & 27,4 & 5,50 & 4,95 & 4,05 & 2,8 & 6,4 \\
\hline 31 & PV & Ilha & 470 & 47,0 & 5,00 & 4,30 & 3,70 & 2,1 & 10,6 \\
\hline 32 & PV & Brusque & 230 & 33,2 & 5,05 & 4,55 & 3,65 & 1,0 & 7,2 \\
\hline 35 & PV & Orleans & 470 & 40,7 & 5,50 & 4,65 & 3,95 & 2,6 & 8,7 \\
\hline 36 & $\mathrm{C}$ & Tubarão & 370 & 29,8 & 5,25 & 4,95 & 4,15 & 7,4 & 14,6 \\
\hline 38 & $\mathrm{CH}$ & Lages & 430 & 66,0 & 5,00 & 5,20 & 4,30 & 6,1 & 15,2 \\
\hline 39 & $\mathrm{CH}$ & Mafra & 390 & 18,8 & 4,75 & 4,90 & 4,30 & 12,4 & 38,6 \\
\hline 40 & $\mathrm{CBH}$ & Irani & 510 & 104 & 4,15 & 3,85 & 3,55 & 1,2 & 27,1 \\
\hline 42 & PVL & Lauro Muller & 430 & 40,3 & 4,20 & 4,15 & 3,50 & 0,8 & 13,4 \\
\hline 44 & $\mathrm{CH}$ & Pouso Redondo & 460 & 41,0 & 4,10 & 4,40 & 3,75 & 7,1 & 19,9 \\
\hline 45 & $\mathrm{C}$ & Rio do Sul & 490 & 35,0 & 4,10 & 3,95 & 3,45 & 2,3 & 15,0 \\
\hline 46 & $\mathrm{HGH}$ & Jundiaí & 600 & 87,0 & 3,95 & 3,90 & 3,60 & 6,0 & 25,6 \\
\hline 48 & $\mathrm{CH}$ & Cerro Alto & 340 & 77,0 & 4,05 & 4,05 & 3,50 & 0,7 & 19,4 \\
\hline
\end{tabular}

${ }^{(1)}$ Dados obtidos por ERNANI \& ALMEIDA (1986).

${ }^{(2)}$ Usou-se a mesma numeração adotada por ERNANI \& ALMEIDA (1986).

${ }^{(3)}$ Soma de bases.

$\mathrm{CH}=$ Cambissolo Húmico;

$\mathrm{C}=$ Cambissolo;

$\mathrm{CBH}=$ Cambissolo Bruno Húmico;

$\mathrm{TR}=$ Terra Roxa Estruturada;

$\mathrm{TB}=$ Terra Bruna Estruturada;

$\mathrm{LR}=$ Latossolo Roxo;

LRH=Latossoro Roxo Húmico;

LBH=Latossolo Bruno Húmico;

LE=Latossolo Vermelho Escuro;

HGH=Glei Húmico;

PV=Podzólico Vermelho Amarelo;

$\mathrm{PE}=$ Podzólico Vermelho Escuro;

PVL= Podzólico Vermelho Amarelo Latossólico. 
solos, de 4,24 para $0,67 \mathrm{cmol}_{\mathrm{c}} \mathrm{kg}^{-1}$. Em nenhum solo ele foi eliminado completamente no $\mathrm{pH} 5,2$, porém em 9 dos 28 solos os valores de $\mathrm{Al}^{3+}$ foram inferiores a $0,5 \mathrm{cmol}_{\mathrm{c}} \mathrm{kg}^{-1}$ e somente 5 solos apresentaram valores superiores a $1,0 \mathrm{cmol}_{\mathrm{c}} \mathrm{kg}^{-1}$. Nesse valor de $\mathrm{pH}$, nenhum solo apresentou $\mathrm{Al}^{3+}$ superior a $1,5 \mathrm{cmol}_{\mathrm{c}} \mathrm{kg}^{-1}$, mesmo aqueles que originalmente apresentavam mais de $6,0 \mathrm{cmol}_{\mathrm{c}} \mathrm{kg}^{-1}$ (tabela 2).

Nesse trabalho, os solos com maior tamponamento possuem também altos teores de matéria orgânica, o que minimiza a toxicidade do $\mathrm{Al}$ aos vegetais (KAPLAND \& ESTES, 1985). Por isso, é importante preservar ao máximo a M.O. dos solos altamente tamponados, principalmente quando o $\mathrm{pH}$ é inferior a 5,5, pois os valores de $\mathrm{Al}$ ligados à M.O. são muito altos (FIGUEIREDO \& ALMEIDA, 1991; ERNANI et al., 1998) e podem ser liberados para a solução do solo quando esta for decomposta (BLOOM et al., 1985). O aumento do teor de $\mathrm{P}$ no solo, garantindo maior mobilidade desse nutriente em direção às raízes, também permite a obtenção de altos rendimentos vegetais na presença de $\mathrm{Al}$, uma vez que as plantas ficam menos dependentes da existência de um amplo sistema radicular (ERNANI et al., 1997).

Os valores de $\mathrm{H}+\mathrm{Al}$ diminuíram, em média, de 13,0 no $\mathrm{pH}$ original dos solos para $8,7 \mathrm{cmol}_{\mathrm{c}} \mathrm{kg}^{-1}$ no $\mathrm{pH} 5,2$, sendo que as amplitudes de variação desses parâmetros foram respectivamente de 4,2 a 27,1 $\mathrm{cmol}_{\mathrm{c}} \mathrm{kg}^{-1}$ e de 2,8 a $21,4 \mathrm{cmol}_{\mathrm{c}} \mathrm{kg}^{-1}$, o

Tabela 2 - Valores de $\mathrm{Al}^{3+}, \mathrm{H}^{+}+\mathrm{Al}^{3+}, \mathrm{Ca}^{++}+\mathrm{Mg}^{++}$, saturação por bases (V\%) e saturação por alumínio (m\%) nas amostras de solo com pH natural (nat.) ou com pH- $\mathrm{H}_{2} \mathrm{O} 5,2$ (pH em $\left.\mathrm{CaCl}_{2} 4,7\right)$. Média de 2 repetições.

\begin{tabular}{|c|c|c|c|c|c|c|c|c|c|c|}
\hline \multirow[t]{2}{*}{ Solo } & \multicolumn{2}{|c|}{$\mathrm{Al}^{3+}$} & \multicolumn{2}{|c|}{$\mathrm{H}^{+}+\mathrm{Al}^{3+}$} & \multicolumn{2}{|c|}{$\mathrm{Ca}^{++}+\mathrm{Mg}^{++}$} & \multicolumn{2}{|c|}{$\mathrm{V}$} & \multicolumn{2}{|c|}{$\mathrm{m}$} \\
\hline & nat. & pH 5,2 & nat. & pH 5,2 & nat. & pH 5,2 & nat. & pH 5,2 & nat. & $\mathrm{pH} 5,2$ \\
\hline 01 & 4,78 & 0,69 & 12,42 & 8,24 & 1,18 & 5,70 & 11 & 42 & 76 & 12 \\
\hline 02 & 4,52 & 0,60 & 10,67 & 7,34 & 4,16 & 6,86 & 29 & 50 & 51 & 8 \\
\hline 03 & 3,72 & 0,61 & 11,70 & 8,44 & 2,34 & 6,37 & 18 & 43 & 59 & 9 \\
\hline 06 & 4,38 & 1,18 & 20,75 & 13,85 & 4,25 & 9,88 & 18 & 43 & 49 & 12 \\
\hline 10 & 3,66 & 0,51 & 16,32 & 10,93 & 1,41 & 6,62 & 9 & 38 & 69 & 9 \\
\hline 13 & 4,54 & 0,74 & 13,59 & 9,63 & 1,95 & 6,53 & 14 & 41 & 68 & 11 \\
\hline 14 & 4,56 & 0,75 & 15,13 & 11,07 & 2,42 & 7,79 & 16 & 42 & 61 & 10 \\
\hline 16 & 5,06 & 0,82 & 15,62 & 10,51 & 3,10 & 8,72 & 18 & 45 & 60 & 10 \\
\hline 17 & 3,76 & 0,21 & 12,69 & 7,19 & 3,84 & 8,03 & 25 & 55 & 47 & 3 \\
\hline 19 & 5,21 & 0,83 & 14,10 & 10,58 & 3,35 & 7,94 & 20 & 44 & 59 & 10 \\
\hline 22 & 2,67 & 0,27 & 11,74 & 8,16 & 1,24 & 6,02 & 11 & 42 & 66 & 6 \\
\hline 23 & 1,12 & 0,21 & 6,26 & 4,84 & 2,99 & 4,59 & 34 & 50 & 26 & 4 \\
\hline 24 & 3,28 & 0,64 & 13,03 & 9,00 & 7,18 & 11,54 & 38 & 57 & 29 & 6 \\
\hline 28 & 1,79 & 0,43 & 6,59 & 4,92 & 1,70 & 4,14 & 36 & 49 & 47 & 10 \\
\hline 29 & 1,26 & 0,26 & 10,57 & 7,58 & 5,78 & 7,33 & 36 & 51 & 17 & 3 \\
\hline 30 & 1,09 & 0,15 & 4,21 & 2,79 & 2,78 & 4,67 & 42 & 62 & 27 & 3 \\
\hline 31 & 3,98 & 0,54 & 8,52 & 5,61 & 2,87 & 5,86 & 27 & 52 & 47 & 9 \\
\hline 32 & 2,59 & 0,25 & 5,71 & 3,86 & 2,97 & 5,45 & 35 & 58 & 45 & 4 \\
\hline 35 & 1,07 & 0,22 & 5,91 & 4,20 & 2,69 & 5,05 & 33 & 54 & 27 & 4 \\
\hline 36 & 2,26 & 0,57 & 10,63 & 8,29 & 7,88 & 10,65 & 43 & 56 & 22 & 5 \\
\hline 38 & 1,93 & 0,47 & 9,87 & 7,32 & 6,62 & 9,89 & 41 & 58 & 22 & 5 \\
\hline 39 & 4,06 & 0,98 & 27,07 & 21,39 & 12,96 & 19,88 & 33 & 48 & 23 & 5 \\
\hline 40 & 7,92 & 1,52 & 19,42 & 13,39 & 1,74 & 8,39 & 10 & 38 & 80 & 18 \\
\hline 42 & 6,45 & 0,70 & 14,76 & 8,08 & 0,20 & 7,81 & 3 & 47 & 94 & 10 \\
\hline 44 & 6,82 & 0,83 & 12,69 & 6,79 & 4,78 & 11,19 & 30 & 63 & 55 & 7 \\
\hline 45 & 8,67 & 1,02 & 13,24 & 6,04 & 1,81 & 9,77 & 17 & 62 & 76 & 10 \\
\hline 46 & 9,85 & 1,31 & 22,23 & 13,41 & 5,30 & 17,69 & 22 & 56 & 62 & 8 \\
\hline 48 & 7,75 & 1,45 & 19,87 & 11,33 & 1,54 & 10,38 & 8 & 48 & 81 & 16 \\
\hline Médias & 4,24 & 0,67 & 13,04 & 8,74 & 3,61 & 8,38 & 24 & 50 & 52 & 8 \\
\hline Variação & $1,07-9,85$ & $0,15-1,52$ & $4,21-27,07$ & $2,79-21,39$ & $0,20-12,96$ & $4,14-19,88$ & $3-43$ & $38-63$ & $17-94$ & $3-18$ \\
\hline
\end{tabular}

Ciência Rural, v. 29, n. 4, 1999. 
que demonstra o alto poder tamponante dos solos utilizados (tabela 2). A magnitude da diminuição foi menor no $\mathrm{H}+\mathrm{Al}$ do que no $\mathrm{Al}^{3+}$, tendo em vista que este último é o principal componente tamponante da acidez até $\mathrm{pH}$ próximo de 5,2, enquanto que o $\mathrm{H}$ tampona a acidez até valores de $\mathrm{pH}$ mais elevados.

A saturação média de alumínio na CTC diminuiu de $52 \%$ para $8 \%$ no $\mathrm{pH} 5,2$. Somente em cinco solos a $\mathrm{m} \%$ foi superior a $10 \%$ nesse valor de $\mathrm{pH}$, e em três desses ela era superior a $75 \%$ no $\mathrm{pH}$ natural. No pH 5,2, nenhum solo apresentou índice $\mathrm{m}$ superior a $20 \%$, o que mostra que a chance de haver toxicidade de $\mathrm{Al}$ para as culturas nesse valor de $\mathrm{pH}$ é bastante limitada. A amplitude de variação da \%m variou de 17 a 94 nos solos com $\mathrm{pH}$ original e de apenas 3 a 18 nos solos com pH 5,2 (tabela 2).

Os valores de $\mathrm{Ca}+\mathrm{Mg}$ do solo aumentaram de 3,6 $\mathrm{cmol}_{\mathrm{c}} \mathrm{kg}^{-1}$ nas amostras sem calcário para $8,4 \mathrm{cmol}_{\mathrm{c}} \mathrm{kg}^{-1}$ nas com $\mathrm{pH} 5,2$, aumentando conseqüentemente a saturação por bases de 24 para $50 \%$ (tabela 2). Esses valores de $\mathrm{Ca}+\mathrm{Mg}$ são muito superiores ao nível crítico atribuído pela Rede Oficial de Laboratórios de Análise de Solos (ROLAS) dos estados do Rio Grande do Sul e de Santa Catarina, que é de $5,0 \mathrm{cmol}_{\mathrm{c}} \mathrm{kg}^{-1}$ (COMISSÃO, 1995). Os incrementos observados nos teores de $\mathrm{Ca}+\mathrm{Mg}$ e saturação com bases, e a diminuição do $\mathrm{Al}$ trocável e da percentagem de $\mathrm{Al}$ na CTC, representam melhorias significativas na fertilidade do solo, que certamente deverão se refletir no aumento da produtividade para a maioria das culturas nesses solos com alto tamponamento. Para esses solos, que têm alta necessidade de calcário, a correção da acidez até $\mathrm{pH}$ 5,2 pode constituir-se numa alternativa econômica viável para os produtores de baixa renda, principalmente no início do processo produtivo. Vários trabalhos mostram bons rendimentos das culturas, mesmo em solos com acidez elevada. QUAGGIO et al. (1998) obtiveram a máxima produtividade de grãos de soja quando o solo apresentava saturação de bases de $50 \%$, na presença de Mo nas sementes. CAIRES et al. (1998) não obtiveram aumento de rendimento de soja pela aplicação de calcário sobre a superfície de um Latossolo Vermelho Escuro que possuía $32 \%$ de saturação de bases, $18 \%$ de saturação com alumínio e pH 4,5. ERNANI et al. (1998) obtiveram aumento de rendimento de milho pela calagem de apenas $17 \%$ num Latossolo Bruno que possuía $\mathrm{pH} 4,7, \mathrm{Al}=3,3 \mathrm{cmol}_{\mathrm{c}} \mathrm{kg}^{-1}$ e $56 \%$ de saturação com Al.

As equações de regressão entre a necessidade de calcário obtida por incubação para elevar o pH em $\mathrm{H}_{2} \mathrm{O}$ a 5,2 com a necessidade fornecida pelos diferentes métodos apresentaram altos coeficientes de correlação (tabela 3). A combinação de $\mathrm{Al}+\mathrm{M}$.O. teve o coeficiente de correlação mais elevado $\left(r^{2}=0,94\right)$, seguido, em ordem decrescente, pelos métodos SMP $\left(\mathrm{r}^{2}=0,91\right), \mathrm{Al}^{3+}$ $\left(r^{2}=0,90\right), H+A l\left(r^{2}=0,88\right)$, e saturação por bases $\left(r^{2}=0,83\right)$. Estes resultados diferem dos obtidos por CIPRANDI (1993) para elevar o $\mathrm{pH}-\mathrm{H}_{2} \mathrm{O}$ dos solos com baixos teores de argila, do Rio Grande do Sul a 5,5 , onde o coeficiente de correlação para o $\mathrm{Al}+$ M.O. foi de 0,73, muito inferior ao do método SMP, que foi 0,84. KAMINSKI (1974) e ERNANI \& ALMEIDA (1986), trabalhando com solos do RS e SC respectivamente, obtiveram coeficientes de correlação maiores para o $\mathrm{H}+\mathrm{Al}$ do que para o índice SMP na determinação da quantidade de calcário para elevar o pH a 5,5, 6,0 e 6,5.

A leitura do $\mathrm{pH}$ SMP, como vem sendo executada nos laboratórios de análise de solos do RS e SC, pode também ser utilizada para estimar a necessidade de calcário dos solos para elevar o $\mathrm{pH}-\mathrm{H}_{2} \mathrm{O}$ até 5,2. Considerando que os teores de $\mathrm{Al}$ e de M.O. também são determinados nas análises de rotina, seus valores podem igualmente ser empregados para conferência das necessidades de calcário dos solos a partir de equação constante na tabela 3 .

As reduções nas quantidades de calcário recomendadas para elevar o pH a 5,2 são, em média, de 1,4 e $3,1 \mathrm{t} \mathrm{ha}^{-1}$ inferiores àquelas necessárias para elevar o pH a 5,5 ou 6,0. As reduções são mais expressivas quando são considerados somente os solos altamente tamponados, com pH SMP inferior a 5,0, nos quais as quantidades recomendadas para $\mathrm{pH} 5,2$ são, em média, 3,0 e 7,0t ha ${ }^{-1}$ inferiores às recomendadas para elevar o $\mathrm{pH}$ a 5,5 ou 6,0. Para solos com pH-SMP 4,4 a economia com calcário atingiria respectivamente 5,0 e $10,6 \mathrm{t} \mathrm{ha}^{-1}$ (tabela 4 ).

Tabela 3 - Equações de regressão e coeficientes de correlação (r) entre as necessidades de calcário ( $\mathrm{t} \mathrm{ha}^{-1}$ ) obtidas pelo método da incubação com as fornecidas pelos métodos do $\mathrm{Al}, \mathrm{H}+\mathrm{Al}, \mathrm{SMP}$, saturação por bases, e $\mathrm{Al}+$ M.O.

\begin{tabular}{lcl}
\hline \multicolumn{1}{c}{ Métodos } & (r) & Equações \\
& & \\
\hline & & \\
$\mathrm{Al}^{3+}$ & 0,90 & $\mathrm{NC}=0,66+2,40 \mathrm{Al}^{3+}-0,102\left(\mathrm{Al}^{3+}\right)^{2}$ \\
$\mathrm{H}+\mathrm{Al}$ & 0,88 & $\mathrm{NC}=-2,99+1,12(\mathrm{H}+\mathrm{Al})-0,020(\mathrm{H}+\mathrm{Al})^{2}$ \\
$\mathrm{SMP} *$ & 0,91 & $\mathrm{NC}=91,31-28,43 \mathrm{SMP}+2,230(\mathrm{SMP})^{2}$ \\
Saturação por Bases (V\%) & 0,83 & $\mathrm{NC}=1,54+1,62 \mathrm{~V} \%-0,055(\mathrm{~V} \%)^{2}$ \\
$\mathrm{Al}^{3+}$ e Matéria Orgânica & 0,94 & $\mathrm{NC}=0,43+1,38 \mathrm{Al}+0,363 \mathrm{M} . \mathrm{O}$. \\
& & \\
\hline
\end{tabular}

* Relação solo/água/solução SMP de 1:1:0,5. 
Tabela 4 - Quantidades de calcário $\left(\mathrm{t} \mathrm{ha}^{-1}\right)$ obtidas pelo método SMP para elevar o $\mathrm{pH}-\mathrm{H}_{2} \mathrm{O}$ dos solos desse trabalho a 5,2 e recomendadas pela Rede Oficial de Laboratórios de Análise de Solos do RS e SC (ROLAS) para elevar o $\mathrm{pH}$ dos solos a três valores de $\mathrm{pH}$.

\begin{tabular}{|c|c|c|c|c|}
\hline \multirow[t]{2}{*}{$\mathrm{pH}$ SMP } & \multicolumn{4}{|c|}{ pH EM ÁGUA A ATINGIR } \\
\hline & 5,2 & 5,5 & 6,0 & 6,5 \\
\hline & & $---\cdot$ & & \\
\hline 4,4 & 9,4 & 15,0 & 21,0 & 29,0 \\
\hline 4,5 & 8,5 & 12,5 & 17,3 & 24,0 \\
\hline 4,6 & 7,7 & 10,9 & 15,1 & 20,0 \\
\hline 4,7 & 6,9 & 9,6 & 13,3 & 17,5 \\
\hline 4,8 & 6,2 & 8,5 & 11,9 & 15,7 \\
\hline 4,9 & 5,5 & 7,7 & 10,7 & 14,2 \\
\hline 5,0 & 4,9 & 6,6 & 9,9 & 13,3 \\
\hline 5,1 & 4,3 & 6,0 & 9,1 & 12,3 \\
\hline 5,2 & 3,8 & 5,3 & 8,3 & 11,3 \\
\hline 5,3 & 3,3 & 4,8 & 7,5 & 10,4 \\
\hline 5,4 & 2,8 & 4,2 & 6,8 & 9,5 \\
\hline 5,5 & 2,4 & 3,7 & 6,1 & 8,6 \\
\hline 5,6 & 2,0 & 3,2 & 5,4 & 7,8 \\
\hline 5,7 & 1,7 & 2,8 & 4,8 & 7,0 \\
\hline 5,8 & 1,4 & 2,3 & 4,2 & 6,3 \\
\hline 5,9 & 1,2 & 2,0 & 3,7 & 5,6 \\
\hline 6,0 & 1,0 & 1,6 & 3,2 & 4,9 \\
\hline 6,1 & 0,9 & 1,3 & 2,7 & 4,3 \\
\hline 6,2 & 0,8 & 1,0 & 2,2 & 3,7 \\
\hline 6,3 & 0,7 & 0,8 & 1,8 & 3,1 \\
\hline 6,4 & 0,4 & 0,6 & 1,4 & 2,6 \\
\hline 6,5 & 0,2 & 0,4 & 1,1 & 2,1 \\
\hline 6,6 & 0,0 & 0,2 & 0,8 & 1,6 \\
\hline 6,7 & 0,0 & 0,0 & 0,5 & 1,2 \\
\hline 6,8 & 0,0 & 0,0 & 0,3 & 0,8 \\
\hline 6,9 & 0,0 & 0,0 & 0,2 & 0,5 \\
\hline 7,0 & 0,0 & 0,0 & 0,0 & 0,2 \\
\hline Médias* & 3,4 & 4,8 & 6,5 & 8,8 \\
\hline
\end{tabular}

* Obtidas somente a partir dos valores onde a recomendação de calcário foi diferente de zero.

\section{CONCLUSÕES}

A elevação do $\mathrm{pH}$ em $\mathrm{H}_{2} \mathrm{O}$ para 5,2 diminuiu o alumínio trocável médio dos solos de 4,24 para $0,67 \mathrm{cmol}_{\mathrm{c}} \mathrm{kg}^{-1}$ e a m\% de 52 para $8 \%$, e aumentou o $\mathrm{Ca}+\mathrm{Mg}$ de 3,6 para 8,4cmol $\mathrm{kg}^{-1}$ e a V\% de 24 para $50 \%$. Todos os métodos testados foram eficientes para recomendar calcário para elevar o $\mathrm{pH}$ em água até 5,2 . A elevação do $\mathrm{pH}$ em $\mathrm{H}_{2} \mathrm{O}$ para 5,2 ao invés de 5,5 ou 6,0 reduziria a quantidade média de calcário a aplicar em 1,4 e $3,1 \mathrm{t} \mathrm{ha}^{-1}$, respectivamente; para os solos mais tamponados, com $\mathrm{pH}$ SMP inferior a 5,0, a redução seria, respectivamente, de 3,0 e $7,0 \mathrm{tha}^{-1}$.

\section{REFERÊNCIAS BIBLIOGRÁFFIAS}

BLOOM, P.R., McBRIDE, M.B., WEAVER, R.M. Aluminum organic matter in acid soils: buffering and solution aluminum activity. Soil Science Society of America Journal, Madison, v. 43 , p. $488-493,1985$.
CAIRES, E.F., CHUEIRI, W.A., MADRUGA, E.F., $\boldsymbol{e} \boldsymbol{t}$ al. Alterações de características químicas do solo e resposta da soja ao calcário e gesso aplicados na superfície em sistema de cultivo sem preparo do solo. Revista Brasileira de Ciência do Solo, Campinas, v. 22, p. 27-34, 1998.

CIPRANDI, M.A.O. Avaliação da metodologia de determinação da acidez ativa e potencial em solos do Rio Grande do Sul. Porto Alegre - RS. 1993. 90p. Dissertação (Mestrado em Ciência do Solo) - Curso de Pós-graduação em Agronomia, Universidade Federal do Rio Grande do Sul, 1993.

COMISSÃO DE FERTILIDADE DO SOLO - RS/SC Recomendação de adubação e calagem para os Estados do Rio Grande do Sul e Santa Catarina. 3. ed. Passo Fundo: SBCS - Núcleo Regional Sul/EMBRAPA/CNPT, 1995. 224 p.

ERNANI, P.R., ALMEIDA, J.A. Comparação de métodos analíticos para avaliar a necessidade de calcário dos solos do Estado de Santa Catarina. Revista Brasileira de Ciência do Solo, Campinas, v. 10, n. 2, p. 143-150, 1986.

ERNANI, P.R., NASCIMENTO, J.A., CAMPOS, M.L. O aumento do fósforo no solo diminui a fitotoxicidade do alumínio. In: XXVI CONGRESSO BRASILEIRO DE CIÊNCIA DO SOLO, 1997. Rio de Janeiro, RJ. Anais... Rio de Janeiro: Soc. Bras. de Ciência do Solo, 1997. (CD-Room).

ERNANI, P.R., NASCIMENTO, J.A.L., OLIVEIRA, L.C. Increase of grain and green matter of corn by liming. Revista Brasileira de Ciência do Solo, Viçosa, v. 22, n. 2, p. 275280,1998

FIGUEIREDO, O.A.R., ALMEIDA, J.A. Quantificação das formas trocáveis e não trocáveis de alumínio em solos ácidos do Estado de Santa Catarina. Revista Brasileira de Ciência do Solo, Campinas, v. 15, n. 2, p. 151-156, 1991.

KAMINSKI, S.J. Fatores de acidez e necessidade de calcário em solos do Rio Grande do Sul. Porto Alegre - RS. 1974.96 p. Dissertação (Mestrado em Ciência do Solo) - Curso de Pósgraduação em Agronomia, Faculdade de Agronomia, Universidade Federal do Rio Grande do Sul, 1974.

KAPLAND, D.I., ESTES, G.O. Organic matter relationship to soil nutrient status and aluminum toxicity in Alfafa. Agronomy Journal, Madison, v. 77, p. 735-738, 1985.

MASCARENHAS, H.A.A. Calagem para soja. In: REUNIÃO BRASILEIRA DE FERTILIDADE DO SOLO, 15., 1983, Campinas. Anais... Campinas: Sociedade Brasileira de Ciência do Solo, 1983. 40 p. p. 145-163.

QUAGGIO, J.A. Resposta das culturas à calagem em outros Estados. In: SEMINÁRIO SOBRE CORRETIVOS DA ACIDEZ DO SOLO, 1989. Santa Maria, RS. Anais... Santa Maria: UFSM, 1983. 224 p. p. 177-199.

QUAGGIO, J.A., GALLO, P.B., FURLANI, A.M.C., et al Isoquantas de produtividade de soja e sorgo para níveis de calagem e molibdênio. Revista Brasileira de Ciência do Solo, Viçosa, v. 22, n. 2, p. 337-344, 1998

SIQUEIRA, O.J.F. Resposta das culturas à calagem e a fósforo em solos do Rio Grande do Sul e de Santa Catarina e seus reflexos técnico-econômicos. In: SEMINÁRIO SOBRE CORRETIVOS DA ACIDEZ DO SOLO, 1983. Santa Maria, RS. Anais... Santa Maria: UFSM, 1989. 224 p. p.151-176.

TEDESCO, M.J., GIANELlO, C., BISSANI, C.A., $\boldsymbol{e t} \boldsymbol{a l}$. Análise de solo, plantas e outros materiais. Porto Alegre: Departamento de Solos, Universidade Federal do Rio Grande do Sul, 1995. 174 p. Boletim Técnico, 5. 